\title{
Outflow-Circumstellar Envelope Interactions in Protostars
}

\author{
Héctor G. Arce \\ California Institute of Technology, Astronomy Department, MS. 105-24, \\ Pasadena, CA 91125
}

\begin{abstract}
We present the results of a high angular resolution $\left(<5^{\prime \prime}\right)$ survey of protostars with outflows, at different evolutionary stages, using the Owens Valley Radio Observatory Millimeter Array. This survey aims to understand the evolution of the interaction between protostellar outflows and the infalling circumstellar envelopes. Our data enable us to probe the structure and kinematics of the molecular outflow and the circumstellar envelope, as well as the outflow-envelope interaction at scales of less than $0.02 \mathrm{pc}$, for every source in our sample. Our results indicate that outflows have a considerable impact on the dense circumstellar envelope. Also, we show that the nature of the outflow-envelope interaction depends on the protostar's evolutionary stage.
\end{abstract}

\section{Introduction}

As stars form inside molecular clouds, they gravitationally gather gas from their surrounding gaseous environment and disk, while at the same time they energetically spurt out vast amounts of mass in a bipolar flow. The stellar outflow originates very close to the star and so it must interact with the gas envelope surrounding the young stellar object (YSO). It is in this region that outflows may have the strongest effect on the star-formation process, as the interaction between the outflow and the accreting envelope could play a crucial role in the mass assembling process and the final mass of the forming star (Adams \& Fatuzzo 1996). The common belief is that stellar outflows widen with time, thereby halting infall and the accreting phase of the protostar. Yet, evidence for outflow cavity widening with time is only seen in one source (Velusamy \& Langer 1998).

Previous studies have shown that outflows strongly interact with their young stars' envelope (e.g., Gueth et al. 1997; Hirano et al. 1997; Hogerheijde et al. 1998). However, it is not yet clear how important outflows are in dispersing the immediate surroundings of the YSO at different stages of the star-formation process, and how they help terminate the infall phase. Here we present the preliminary results of an observational study geared towards understanding the evolution of the outflow-envelope interaction in forming stars. 


\section{Observations}

The observed source list consists of nine young stellar objects at different evolutionary stages (see Figure 1). The sample includes three Class 0 sources (protostars in their youngest evolutionary stage), three Class I sources (which are more evolved than Class 0 sources), and three Class II sources (which are the most evolved sources in our sample).

We observed different molecular lines towards our target sources which trace different density and kinematic regimes. The ${ }^{12} \mathrm{CO}(1-0)$ line was observed in order to study the (low-density) outflow gas. The $\mathrm{C}^{18} \mathrm{O}(1-0)$ line is used to study the inner denser regions of the circumstellar envelope, and the ${ }^{13} \mathrm{CO}(1-0)$ line is used to probe the effects of the outflow in the outer (less dense) parts of the envelope. We also observed the molecular lines $\mathrm{HCO}^{+}(1-0), \mathrm{HNC}(1-0)$, $\mathrm{H}^{13} \mathrm{CO}^{+}(1-0)$ to help us probe the infall motions of the dense circumstellar envelope. With these observations we can study the morphology and kinematics of the molecular outflow and of the circumstellar envelope. In addition to the spectral lines mentioned above, we also obtained $2.7 \mathrm{~mm}$ and $3.4 \mathrm{~mm}$ continuum emission data for each source. All observations were carried out at the Owens Valley Radio Observatory Millimeter Array.

\section{Results}

\subsection{Outflow morphology evolution}

In Figure 1 we show the ${ }^{12} \mathrm{CO}(1-0)$ outflow (redshifted and blueshifted lobes) for all the observed sources. It is evident, from Figure 1, that no outflow is alike. All sources show blueshifted and redshifted molecular gas emission, but the lobe structure of each outflow is strikingly different from other outflows in our sample. Yet, even though the outflows are very different from each other, there are some noticeable trends in our sample.

In Figure 1 it can be seen that there is a trend in the outflow morphology with source evolutionary stage. The youngest protostellar sources in our sample (the Class 0 sources, shown in the top row of panels in Fig. 1) tend to have very collimated molecular outflows. The molecular outflows from the Class I sources in our sample (middle row of panels in Fig. 1) show a wide-angle morphology. And, the Class II sources (bottom row of panels in Fig. 1) have molecular outflows with a messy and clumpy structure. One way to explain such trend is that the outflow cavity widens - and it is able to push more cirmcumstellar gas away from the nascent star- as the source evolves. Thus, by the time the outflow source reaches the Class II stage, we are only able to detect outflowing $\mathrm{CO}$ in the few regions around the protostar where there is any molecular gas left. Below we give further support to this hypothesis.

\subsection{Outflow-envelope interactions in Class 0 sources}

In order to demonstrate the impact of outflows on the circumstellar envelope in Class 0 sources, we concentrate our discussion using the results from our observations of the Class 0 source RNO43. The blueshifted lobe of the RNO43 molecular outflow (see Fig. 1) shows a very collimated, jet-like, morphology. 

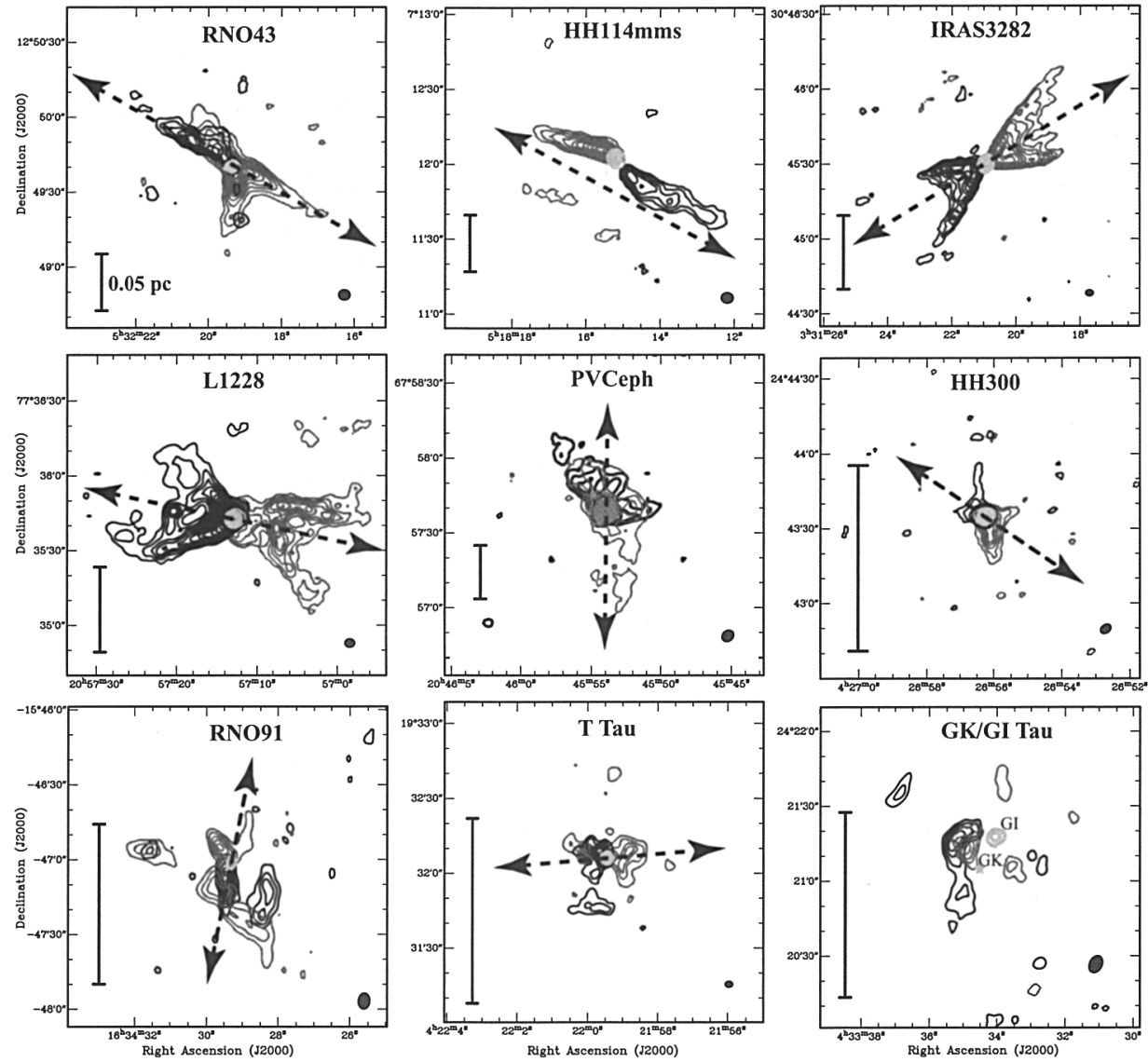

Figure 1. Gallery of ${ }^{12} \mathrm{CO}(1-0)$ outflows in our sample. Black (dark grey) contours show the blueshifted (redshifted) lobe. Light grey contours show the $2.7 \mathrm{~mm}$ continuum emission. The top row of panels shows the three Class 0 sources in our sample, the middle row shows the Class I sources, and the bottom row shows the Class II sources. The synthesized beam of each map is shown on the lower right corner of each panel. The scale in each panel shows the equivalent of $0.05 \mathrm{pc}$ at the assumed distance of each source. The arrow in each panel shows the presumed outflow axis. The name of each outflow is also shown. 

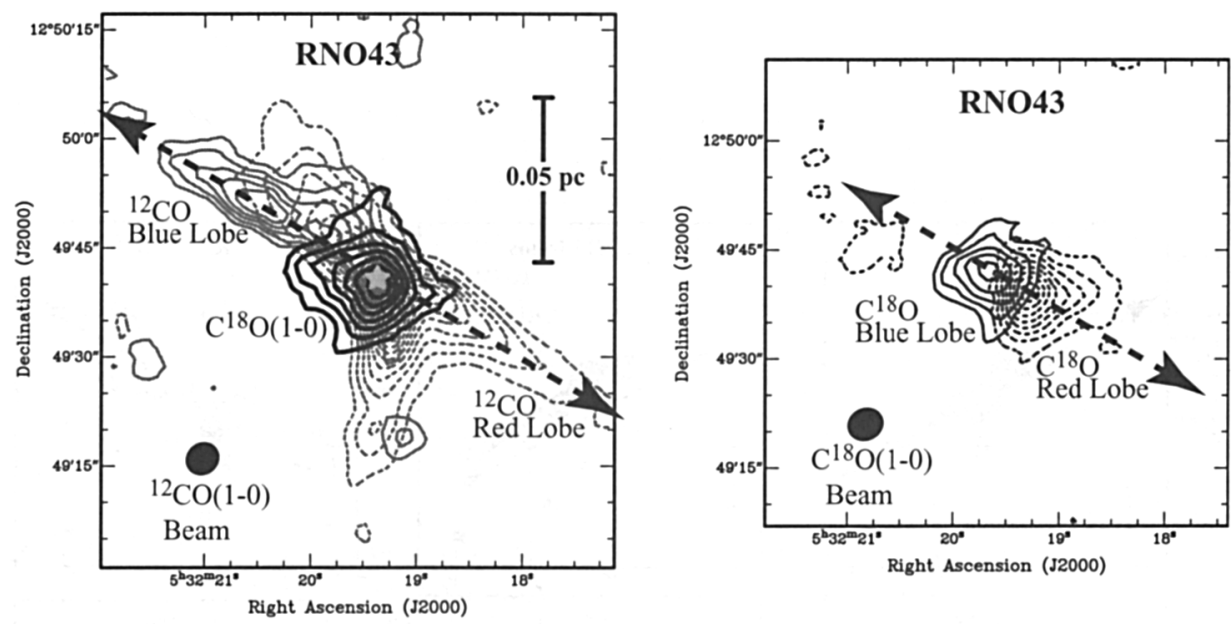

Figure 2. Left. $\mathrm{C}^{18} \mathrm{O}(1-0)$ integrated intensity (black contours) superimposed on the blueshifted (solid gray contours) and redshifted (dashed gray contours) lobes of the RNO43 ${ }^{12} \mathrm{CO}(1-0)$ outflow. The gray star symbol denotes the position of the outflow source. The arrow shows the ${ }^{12} \mathrm{CO}$ outflow axis. Right. $\mathrm{C}^{18} \mathrm{O}(1-0)$ outflow in RNO43. The solid (dashed) contours denote the blueshifted (redshifted) emission. The arrow shows the ${ }^{12} \mathrm{CO}(1-0)$ outflow axis.

The same is expected for the opposite (redshifted) lobe, and thus we are certain that the outflow axis is that shown in Figure 1. We believe that the redshifted emission directly south of the source is due to another outflow from a suspected binary companion.

In Figure 2 (left panel) we plot the integrated intensity (over all velocities) of the $\mathrm{C}^{18} \mathrm{O}(1-0)$ emission surrounding $\mathrm{RNO} 43$. The $\mathrm{C}^{18} \mathrm{O}$ contours show a condensation surrounding the Class 0 source, with the peak intensity coincident with the position of the protostar. Hence, we are convinced that the $\mathrm{C}^{18} \mathrm{O}$ emission probes the circumstellar envelope of RNO43. In the left panel of Figure 2 it can also be seen that a significant area of the $\mathrm{C}^{18} \mathrm{O}$ envelope is coincident with the ${ }^{12} \mathrm{CO}(1-0)$ outflow, suggestive that a considerable amount of envelope material is being affected by the outflow.

In Figure 2 (right panel) we show the $\mathrm{C}^{18} \mathrm{O}$ blueshifted and redshifted emission. It is clear that the contours define a blue lobe and a red lobe in the same sense as, and with an axis similar to that of, the ${ }^{12} \mathrm{CO}(1-0)$ outflow. This strongly suggests that the velocity gradient in the circumstellar envelope traced by the $\mathrm{C}^{18} \mathrm{O}$ emission (i.e., the $\mathrm{C}^{18} \mathrm{O}$ outflow) was caused by the interaction of the RNO43 outflow and the surrounding dense ambient medium. Moreover, if we compare the kinetic energy of the $\mathrm{C}^{18} \mathrm{O}$ outflow and the gravitational binding energy of the very dense circumstellar envelope - traced by the $\mathrm{H}^{13} \mathrm{CO}^{+}(1-0)$ emission - we see that these two energies are about the same $\left(\sim 10^{42} \mathrm{erg}\right)$. Therefore, we see that not only has the RNO43 outflow already put in motion dense gas surrounding the protostar, it also has the required energy to eventually 
push away, clear and potentially gravitationally unbind the RNO43 circumstellar envelope.

\subsection{Outflow-envelope interactions in Class I sources}

In this section we turn our attention to the outflow-envelope interaction in Class I sources. For this purpose, we use the results of our observations of the Class I source L1228.

The ${ }^{12} \mathrm{CO}(1-0)$ outflow from L1228 is shown in Figure 3 (left panel). The most striking feature of this molecular outflow is the well-defined wide-angle morphology of both redshifted and blueshifted lobes. The contours in each lobe seem to delineate the (limb-brightened) walls of parabolic outflow cavities. With only the ${ }^{12} \mathrm{CO}(1-0)$ data we may speculate that such wide-angle outflow cavities will restrict the infalling material to a limited volume around the protostar. Hence, it is possible to learn something about the outflow-envelope interaction by just studying the outflow morphology.

However, with our data of L1228 we can do better than "just speculate". In the middle panel of Figure 3 we show the integrated intensity of the $\mathrm{HCO}^{+}(1-0)$ in the region surrounding the $\mathrm{L} 1228$ outflow source. It is clear that the $\mathrm{HCO}^{+}(1-$ $0)$-a high-density tracer with a critical density of $\sim 2 \times 10^{5} \mathrm{~cm}^{-3}$ - is mainly concentrated outside the outflow boundaries. Thus, we may conclude that the outflow cavities are constraining the volume of the dense circumstellar envelope (as we had speculated using only the outflow morphology).

In addition to the morphology of the circumstellar envelope, our data enables us to also study its kinematics. In regions surrounding young stellar objects the $\mathrm{HCO}^{+}(1-0)$ line is reasonably optically think and may be used to study the infall kinematics in circumstellar envelopes. In order to conduct a proper infall study using millimeter spectral lines an optically thin line is also needed (see, e.g., Evans 1999). In our case we use the optically thin line $\mathrm{H}^{13} \mathrm{CO}^{+}(1-0)$. In Figure 3 (right panel) we show the $\mathrm{HCO}^{+}(1-0)$ and $\mathrm{H}^{13} \mathrm{CO}^{+}(1-0)$ spectra in the region surrounding the $\mathrm{L} 1228$ outflow source. It can be seen that the $\mathrm{HCO}^{+}(1-0)$ line does not show the "prototypical" double-peaked infall profile (see Evans 1999 for details on infall profiles). Instead, the $\mathrm{HCO}^{+}(1-0)$ line shows single-peaked, blue-skewed (relative to the optically thin line) profiles. Such a blue-skewed (single-peaked) profile has been seen in other interferometric $\mathrm{HCO}^{+}(1-0)$ observations of infall candidates (e.g., Choi, Panis, \& Evans 1999). Thus, we are convinced that our $\mathrm{HCO}^{+}(1-0)$ data of $\mathrm{L} 1228$ shows evidence of infall motions in the dense envelope surrounding the protostar.

In summary, our observations of the L1228 outflow source reveal that this Class I protostar is powering an outflow with a clear wide-angle morphology. In addition, the morphology and the kinematics of the $\mathrm{HCO}^{+}(1-0)$ emission are consistent with a picture where the outflow has constrained the infall region to a limited volume outside the outflow lobes. And, it is possible that if the outflow keeps widening it could eventually halt the infall process.

\section{Summary and Conclusions}

Preliminary results from an observational study of nine molecular outflows from young stellar objects at different evolutionary stages show clear trends in the 


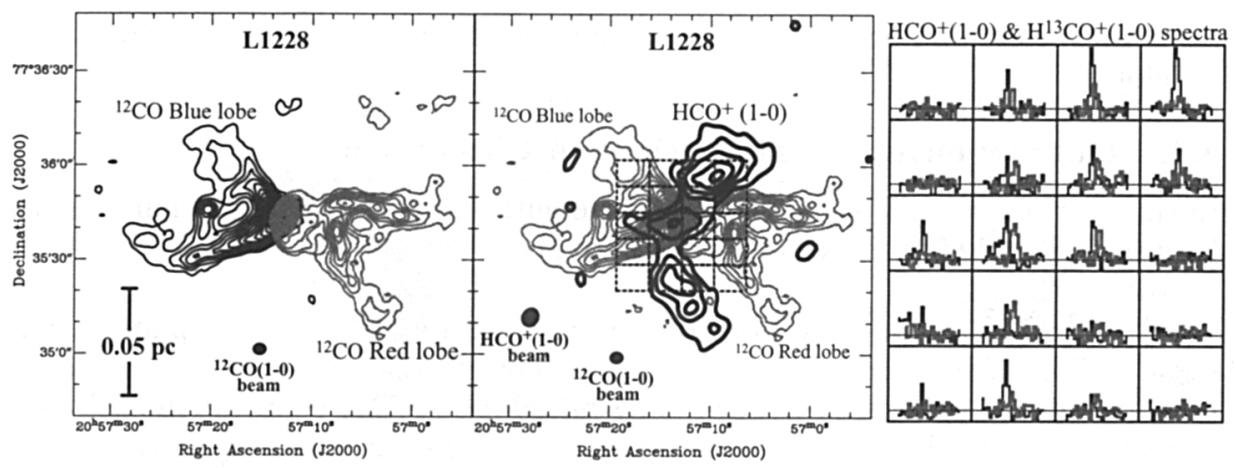

Figure 3. Left. Contour map of the $\mathrm{L} 1228{ }^{12} \mathrm{CO}(1-0)$ outflow. The black contours show the blueshifted lobe and the grey contours show the redshifted lobe. Middle. Integrated intensity map of the $\mathrm{HCO}^{+}(1-$ 0 ) in L1228 (black contours), superimposed on the ${ }^{12} \mathrm{CO}(1-0)$ outflow map (grey contours). Right. $\mathrm{HCO}^{+}(1-0)$ (black) and $\mathrm{H}^{13} \mathrm{CO}^{+}(1-0)$ (grey) spectra in the region indicated by the grid in the middle panel.

outflow-circumstellar envelope interaction. The youngest sources show very collimated outflows, the slightly more evolved sources show outflows with a wide-angle morphology, and the most evolved sources in our sample show a clumpy mess in their molecular outflow structure. Our results also show that the youngest protostars are able to accelerate the dense circumstellar envelope gas to high velocities, and that the wide outflow lobes of the more evolved sources constrain the infalling material to a limited volume around the protostar. We conclude that protostellar outflows have a major impact on the circumstellar envelope of nascent stars, and that they play a crucial role in the the mass assembling process of forming stars.

\section{References}

Adams, F. C., \& Fatuzzo, M. 1996, ApJ, 464, 256

Choi, M., Panis, J.-F., \& Evans, N. J. 1999, ApJS, 122, 519

Evans, N. J. 1999, ARAA, 37, 311

Gueth, F., Guilloteau, S., Dutrey, A., \& Bachiller, R. 1997, A\&A, 323, 943

Hirano, N., Kameya, O., Mikami, H., Saito, S., Umemoto, T., \& Yamamoto, S. 1997, ApJ, 478, 631

Hogerheijde, M. R., van Dishoeck, E. F., Blake, G., \& van Langevelde, H. J. 1998, ApJ, 502, 315

Velusamy, T., \& Langer, W. D. 1998, Nature, 392, 685 